\title{
Frontières
}

\section{Composer avec la mort}

\section{Alain Médam}

Volume 20, numéro 2, printemps 2008

Les musiques et la mort

URI : https://id.erudit.org/iderudit/018356ar

DOI : https://doi.org/10.7202/018356ar

Aller au sommaire du numéro

Éditeur(s)

Université du Québec à Montréal

ISSN

1180-3479 (imprimé)

1916-0976 (numérique)

Découvrir la revue

Citer cet article

Médam, A. (2008). Composer avec la mort. Frontières, 20(2), 100-106. https://doi.org/10.7202/018356ar d'utilisation que vous pouvez consulter en ligne.

https://apropos.erudit.org/fr/usagers/politique-dutilisation/ 


\section{Composer avec la mort}

\section{Alain Médam, \\ sociologue, essayiste, peintre.}

Les musiciens face à la mort? Face à face, affrontement, qu'il faut savoir saisir en l'instant où cette mort qui s'approche met la musique elle-même en question; en l'instant, également, où la musique - pardelà le musicien - interroge cette mort qui s'avance.

Et comment saisir mieux ce point de crucialité qu'en considérant ces œuvres ultimes, terminales, que les musiciens composent au soir de leur existence? Là, tout se condense: tout s'intensifie; tout est décisif. Ce n'est point en tant qu'euxmêmes - en tant qu'individus ordinaires, pris dans la vie courante - que les musiciens s'investissent en ces œuvres. C'est en tant que créateurs. Ce qui meurt, alors - redoutent-ils -, ce n'est pas seulement l'homme qui compose; ce n'est pas cet homme qui, circonstanciellement, se trouve être musicien. Ce qui meurt, ce qui disparaît, c'est le compositeur en l'homme; c'est le musicien que porte en lui, intrinsèquement, cet homme qui s'éteint.

Prisme des œuvres ultimes, donc. De ce que des musiciens, à travers les siècles - à tort ou raison - ont cru être leurs œuvres ultimes, ou bien encore de ce qu'ils composèrent, incertains des jours qui leur restaient, pressentant pourtant leur fin prochaine. À quoi ressemblent-elles, ces œuvres? À des sortes de vaisseaux, pour des passages vers l'inconnu? À des transitions? Des mouvements? Ou plutôt, à des formes de résistance? À des interpositions? À des boucliers? Mais peut-être, encore, faut-il les entendre comme des culminations. Des parachèvements. Des récapitulations. Et peut-être, aussi, doit-on les recevoir comme des dépouillements. Des abandons. De remarquables inachèvements.
Pour esquisser quelques réponses à ce propos, on doit s'attarder aux derniers jours de quelques musiciens. Considérer ces jours ultimes, non point - si possible au plan de l'anecdote; de la biographie événementielle. Un musicien, après tout, si génial qu'il soit, n'est guère qu'un individu comme un autre. C'est au plan de son œuvre, plutôt - en s'attachant à son effort ultime de création, à son désespoir de ne pouvoir œuvrer si haut, si loin qu'il voudrait, à son désir de boucler la boucle, de saluer une dernière fois cette vie qui s'éloigne, à son besoin de provoquer cette inconnue qui, déjà l'étreint -, c'est au plan de son œuvre, donc, qu'il faut savoir saisir le sens du face à face.

1748. Bach est un vieil homme, désormais. Il est malade, quasiment aveugle, usé. Depuis quelques mois, cependant, il a entrepris la composition de l'Art de la Fugue. Ce qu'il voudrait, en cette œuvre la dernière, peut-être, se dit-il -, c'est pouvoir récapituler tout son art de composition; le porter à son paroxysme; à sa perfection. Très peu de thèmes, ici, mais développés longuement en multiples spirales, toujours presque identiques et toujours différentes, s'en allant imperturbablement devant elles, déployant les axiomes d'une vaste démonstration mathématique.

Et cependant que l'homme se décompose - cependant qu'il compose, en cet Art de la Fugue, ce qu'il voudrait entendre comme sa propre intangibilité -, il interpose cette œuvre entre sa fin prochaine et lui. Elle lui permet de résister. C'est ce qu'observe Kundera. Cette composition, note-t-il - parmi toutes les orientations présentes dans l'ensemble de la création musicale de Bach, tout au long de son existence - est celle qui « correspond à la tendance la plus archaïque, la plus étrangère à son époque» (Kundera, 1991).
Pour quelle raison? Pour aller à rebours, précisément! Pour se réfugier - c'est possible - dans les formes imperturbables de la pure polyphonie; des axiomes primordiaux et fondateurs. Pour s'opposer au goût du jour; aux séductions et légèretés des temps nouveaux. Pour refuser les complaisances; les facilités.

Donc, le compositeur conserve. Cette récapitulation est également une résistance; cette souveraine affirmation est une dénégation de ce qui lui échappe. Mais il est une autre dimension, encore, de cette œuvre: elle est un véhicule pour un passage vers un ailleurs; un au-delà. Bach s'implique personnellement. Il est du voyage. Il compose l'Art de la Fugue à partir de son propre nom; à partir de l'équivalence numérologique de celui-ci. En somme, il se met dans le vaisseau. Il est le vaisseau. Il transmigre vers la mort, porté par les courbes de son propre chant.

Mais, à nouveau, une opposition apparaît. Bach s'en va, en effet, il transmigre, mais à rebours. En contre-posture. C'est en «contre-sujet» du patronyme de Bach que se développe l'Art de la Fugue. Sa signature spirituelle se trouve là, présente, en cette œuvre terminale, mais sous forme inversée. En contre-figure. Comme une parade, en quelque sorte. Comme une dénégation - là, à nouveau - de ce à quoi le compositeur s'affronte: cette disparition prochaine, inéluctable, de celui qui porte ce nom.

Or cette œuvre reste inachevée! Nous sommes en 1748, donc, et Bach écrit une dernière page de l'Art de la Fugue qui soudain s'arrête, se suspend dans le vide en un silence d'à tout jamais. Cependant, le compositeur va vivre deux années encore. Il ne s'éteindra qu'en 1750, après avoir eu la force - incapable d'écrire, lui-même - de dicter à son gendre un choral: «Seigneur, me voici devant ton trône». 
Il laisse sa cathédrale, ainsi - toute cette architecture sublime de son Art de la Fugue - dans l'attente infinie d'un parachèvement. Et là encore, pour quelle raison? Pour que cet instant terminal ne se termine pas? Pour que par cet inachèvement, l'œuvre se proclame comme terminale? Nous l'ignorons. Bach, sans doute, l'ignorait-il, lui-même. Ce que nous savons, seulement, c'est ce que nous ressentons; ce que nous éprouvons. Soudain, en effet (et l'effet est le même, à chaque écoute), un dernier cercle - une toute dernière boucle dans une longue suite de développements en spirale - se brise. Le silence prend sa place, tout comme - sur la page de la partition - on voit le blanc de l'infini prendre la place de l'encre de l'écriture humaine. C'est fini. Nous entendons le son d'une disparition. Le son même de l'absence. Le son d'une présence, peut-être, annoncée par l'absence. Bach, quelques mois plus tard, sur son lit de mort, ne dira-t-il pas: «Je vais enfin entendre la véritable musique!»

Septembre 1791. Mozart est nimbé de gloire. Il triomphe. On vient de créer la Flûte enchantée. Il est à l'apogée de son art. Il a mis, dans cet opéra, ce qui lui tient le plus à cœur: l'opposition des lumières aux forces obscurantistes, politiques, religieuses, incarnées par la Reine de la Nuit. Et comme il en va des rites initiatiques de la franc-maçonnerie, on voit deux adolescents prendre conscience d'eux-mêmes, peu à peu, à travers une succession de voyages et d'épreuves de formation. Jamais, comme en cette œuvre, Mozart ne s'est engagé à ce point. Elle est un manifeste. Une proclamation. Une affirmation.

Or, peu de temps auparavant - en juillet 1791 -, tandis qu'il achève l'écriture de la partition de la Flûte, Mozart a reçu la visite d'un messager inconnu, anonyme, qui lui passe commande d'un Requiem. Le compositeur hésite. Il a besoin d'argent. Mais ses convictions antireligieuses le portent à refuser. Pourrait-il concilier son art et ses convictions? Composer comme une messe maçonnique? Finalement, il accepte, décidant de se mettre à l'épreuve de ce genre musical. Son consentement est un défi.

De juillet à novembre 1791, cependant, la santé de Mozart se met à décliner. Des pressentiments l'assaillent. Ce Requiem, est-ce pour lui-même qu'il le compose? Temps d'angoisse! Il en a la certitude, peu à peu: cette maladie qui l'étreint est mortelle. Il n'en sortira pas vivant. C'est ainsi, durant ces sombres mois, qu'il compose le Requiem: qu'il tente, du moins, de le mener à bien, y pensant tout le temps sans pouvoir s'y mettre; préoccupé par celui-ci, presque obsessionnellement, sans parvenir à s'en occuper. S'il s'y consacrait entièrement, n'accélèrerait-il pas son pro-

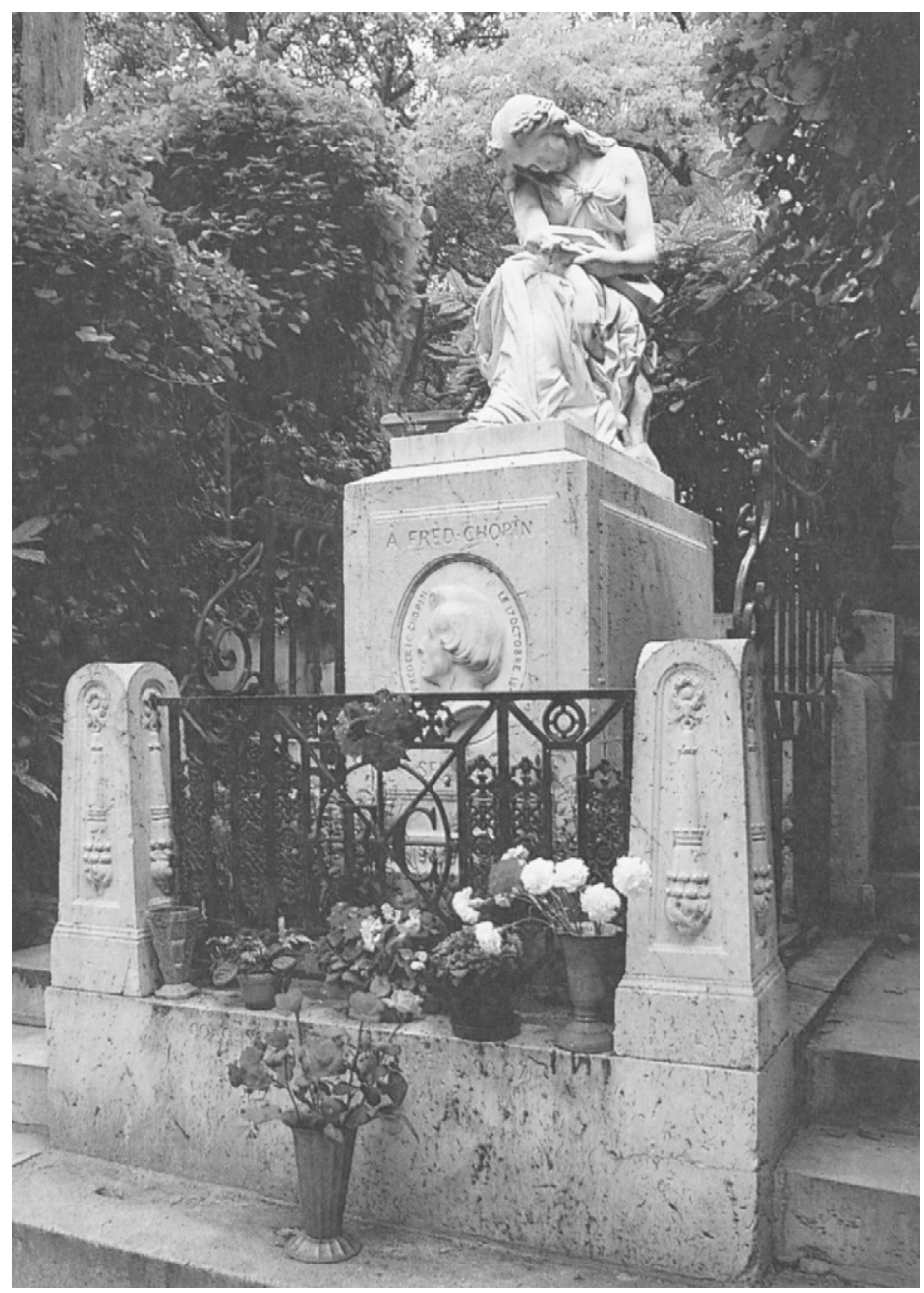

A. CLESINGER, La Musique en pleurs, tombe de CHOPIN, 1850, marbre, cimetière du Père-Lachaise, Paris, France.

s'est-il éteint en lui? Quelle est cette première mort avant la mort? Période d'atonie. De dépression. De stérilité. En marge d'un de ses carnets, le compositeur note nerveusement: «Apprend à te taire!» Mais a-t-il besoin d'apprendre? Cela s'impose à lui : ce silence de la création l'envahit, le paralyse, l'assourdit. N'a-t-il plus qu'à attendre, en silence, le silence de la fin?

En vérité, sous ce vide, se poursuit un travail. Une maturité est à l'œuvre par en dessous. Toute une renaissance se prépare. Soudainement, en effet, tout repart en 1819: de nouvelles compositions; un nouveau langage. Comme si le compositeur avait préparé, dans

pre passage? S'il s'en allait au bout de ce Requiem, n'est-ce pas son oraison funèbre qu'il composerait?

Mozart progresse et se retient, se lance et s'arrête. Finalement, il écrit ce Requiem, mais le laisse inachevé. C'est qu'il a accepté de composer, en ses tout derniers jours, une cantate maçonnique et un lied destinés à célébrer l'inauguration d'un nouveau lieu de réunion de sa Loge. Euvres mineures, certes, mais rassurantes pour lui. Ne revient-il pas à lui-même; à ses convictions et ses amitiés? Les titres de ces deux œuvres, du reste, le disent bien : Enlaçons nos mains et Éloge de l'amitié. La musique de Mozart, ici, retrouve les accents de la Flûte. Comme si le compositeur, à tout prix - par défi? par pudeur? - avait souhaité oublier la mort, ses angoisses, afin de chanter un instant encore l'espérance de la vie. L'inachèvement du Requiem, en ce sens, prend figure d'accomplissement. Il est le prix qu'il faut payer pour que la vie se poursuive, toujours, par-delà quelque mort qu'il soit.

1818. Depuis 1813, Beethoven ne compose presque plus. Rien en 1817. Rien en 1818. Quelque chose, une fois pour toutes, la souffrance de son néant, cette entière mutation stylistique qui le rapproche de lui, de son authenticité; la Neuvième est écrite. La Missia Solomnis. Les dernières sonates. Les derniers quatuors pour corde. La Grande Fugue. Le mouvement se poursuit, ainsi, jusqu'en 1826 - à la veille de la mort -, cependant que la surdité du compositeur s'exaspère, le forçant à s'ouvrir sur son intériorité, sur son être profond, ne tenant plus compte désormais des figures de style convenues, allant toujours plus loin dans ses audaces, en cette quête de l'inouï que sa surdité lui impose.

Il n'était pas mort, donc. Le compositeur, en lui, ne s'était pas éteint. Sans doute, fallait-il que ce passage existe que Beethoven vive, vivant, sa mort de compositeur - pour que le compositeur, en lui, renaisse. Et cette renaissance est presque ésotérique: enfouie au plus profond de l'être. Cet en deçà est un au-delà ; cet enfouissement est un outrepassement. Boucourechliev l'exprime clairement. C'est la force de Beethoven, écrit-il: "se vivre dans sa musique, aux limites. Et se détourner sans cesse du moi sensible vers l'audelà du moi »(Boucourechliev, 1991). 
Mais le moi sensible se rappelle à l'ordre. Beethoven n'y échappe pas. Il est perclus de rhumatismes. Outre cette surdité qui l'accable et l'enferme, il est constamment malade. Il voit mal. Il souffre du foie. Cet état de misère physique s'aggrave mois après mois. Il n'a pourtant que 54 ans! Mais il est vieux. Son neveu, qu'il aime comme son fils, se révolte contre lui. Il s'en désespère. C'est son drame. Il poursuit la lutte, cependant. En dépit du corps et de ses troubles. En dépit du cœur et de ses affections. Quelle lutte? Celle de la création. Elle lui sert à tenir. Chaque œuvre est un combat. Un défi. Boucourechliev, à nouveau: la Grande Fugue, note le musicologue, "est un défi, un ultime cri de guerre» (Boucourechliev, 1991).

Guerre contre qui? Contre la mort? Tout contre la mort, au nom de la vie? Le tout dernier Quatuor pour cordes - opus 135 -, composé à la veille de la fin dernière, en plein drame avec ce neveu qui le tourmente, exprime pourtant une grande paix de l'âme; une sublime sérénité. C'est qu'au cours de cet affrontement terminal se dédoublent les réalités. Un Beethoven meurt, certes - celui du corps et de la chair - cependant qu'un autre Beethoven se refuse à disparaître: celui de l'âme et de la volonté. La musique de Beethoven exprime ce dédoublement. Quel est donc, se demande Boucourechliev (1991), ici, «ce compositeur inconnaissable autrement que par chacune de ses œuvres»? Et plus loin, le musicologue insiste: «Qui est donc le Beethoven des quatuors et des sonates? L'œuvre, seule, nous le dévoile. C'est elle qui nous parle de cet autre moi que l'artiste s'est donné par l'acte même de l'écriture, cet autre moi en quoi s'est transcendé son moi quotidien.»

Seul va disparaître le moi quotidien. L'autre moi - et cela Beethoven le sait, Beethoven le veut - ne peut disparaître. Ces œuvres terminales - qui sont un adieu - sont celles par lesquelles, également, s'engendre l'existence de ce moi qui ne disparaît pas.

1854. Depuis un an déjà, Schumann est en proie à des hallucinations auditives. Incessantes, elles l'accablent. Désormais, jusqu'à sa mort, en 1856, elles ne le quitteront plus. Pour l'instant, il ignore cela : il parle d'une musique qui l'habite, qui l'obsède, sublime et atroce en même temps, une musique devenue folle et qui le mène lui-même - il en est sûr - à la folie. Il ne compose plus. Se détache du monde. S'éloigne des siens. Le 26 février 1854, ne supportant plus cette déchéance dont il est conscient, il demande à être interné. Le lendemain, il se jette dans le Rhin. On le sauve. Mais c'est une épave qu'on tire de l'eau.
L'eau, donc... Quelques jours auparavant, il le confiait: "Je me noie dans les sons », disait-il. Et voici qu'à présent, plutôt que dériver vers la folie, il se précipite dans ce fleuve qui, depuis toujours, l'a fasciné, "fleuve gothique de la Symphonie Rhénane», note Michel Schneider, qui poursuit par ces mots: «ce n'est que dans l'eau devenue musique qu'il pouvait espérer cesser d'entendre l'innere Stimme de la mort, en ne faisant plus qu'un enfin avec elle» (Schneider, 1989).

Musique fluide, glissante, fuyante, envahissante, Schumann sauvé des flots se retrouve englouti dans les eaux de cette musique qui le submerge et qu'il tente désespérément (malgré elle, en somme) de transformer en «vraie» musique, composée de notes consignées dans une partition. Mais c'est impossible! Peu de jours avant sa tentative de suicide, il s'y était essayé pourtant, composant cette œuvre ultime: Variations sur le thème des esprits, dite aussi: "Variation fantômes». C'est sous la dictée, soutenait Schumann, d'esprits "surgis dans la nuit comme des fantômes ", qu'il l'avait composée. D'autres jours, il affirmait que c'était «sous la dictée des anges » qu'elle était sortie de ses mains. Et ces variations sont là, désormais, témoignant de la désespérance de leur auteur. À leur propos, Michel Schneider parle d'un «langage musical fait d'éléments disjoints». Il précise: "Une musique qui se veut si totalement sans limite, qu'elle en devient presque nulle. Elle est absolument, épouvantablement»(Schneider, 1989).

Plus tard, Clara Schumann voudra détruire cette partition. Déchirer ce par quoi son amour fut déchiré. Démolir cette folie restée là, devenue dérision de musique. Que cette chose, surtout, ne puisse être jouée! Qu'elle meure! Disparaisse! Mais cette œuvre subsiste. Elle nous est restée. Et ce qu'on entend lorsqu'on l'écoute, c'est l'assourdissement d'un corps à corps. Le compositeur se débat, se déchire dans sa musique. Il se blesse, se bat contre elle pour pouvoir exprimer par-delà les formes qu'elle lui impose - ce qu'il souffre et ne parvient à dire. La musique - et Schumann - ne s'en sortent alors plus. Crient à l'aide. Appellent désespérément pour qu'on les sorte du trou où ils s'enfoncent, tous deux. Enfin, sur la toute fin dernière semble percer un sourire; comme une acceptation, peut-être, de ce qui ne peut plus guère qu'advenir. Et tout s'achève, s'échoue enfin dans le silence, en un grand vide d'illuminé.

Jusqu'au jour de sa mort, en mai 1856 , Schumann demeurera enfermé à l'asile d'Endenich. Un piano sera à sa disposition. Il y jouera de temps à autre, mais « comme un forcené» affirment plusieurs témoins.

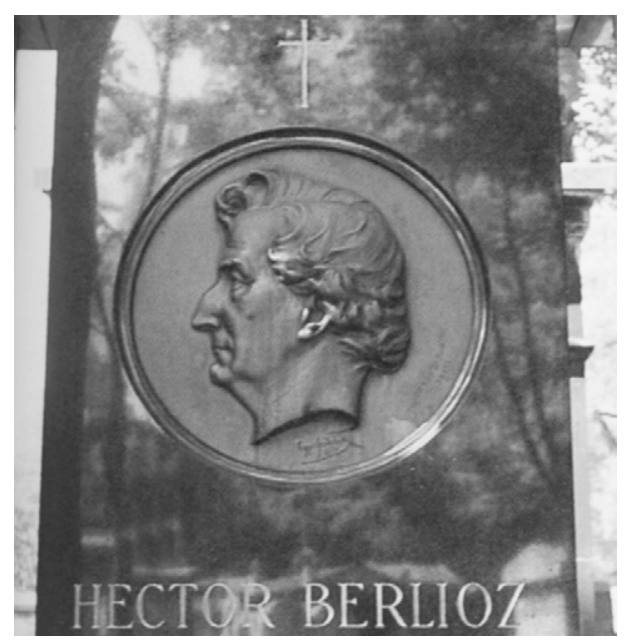

Cyprian GODEBSKI, Médaillon en bronze ornant le monument d'Hector Berlioz (1803-1869)

Poussant des cris. Gesticulant, lui-même, comme pour accompagner ses gesticulations sonores. Bat-il des bras, ainsi, pour se sortir encore, toujours, d'une eau où il s'enfonce? Comble-t-il un vide à tout prix, par ces fracas qui ne sont que silences finalement, à la façon des sons d'un cauchemar?

1894. Pourquoi Bruckner s'est-il arrêté de composer sa Neuvième Symphonie? À jamais, elle restera ainsi: dépourvue de finale. Déjà, avant d'entreprendre son écriture, le compositeur avait hésité. Une Neuvième! Beethoven n'était-il pas mort après sa Neuvième? Bruckner est un homme simple, pieux; sa naïveté est proverbiale dans les sphères cultivées de la Vienne de l'époque. Il se retient, donc. Puis se décide. Il faut qu'il y passe, en quelque sorte. Peut-être en sortira-t-il sans dommage. Peut-être, non. Il n'a pas le choix! S'il s'arrêtait là où il en est, de sa musique, c'est elle qu'il mettrait à mort. Il la tuerait pour pouvoir vivre. Mais en se soumettant à elle, à sa force vitale, c'est lui-même qu'il condamne, redoute-t-il.

Double contrainte. Double péril. Entre les deux, Bruckner compose. Trois mouvements se suivent, le troisième, un adagio, a déjà les accents d'un parachèvement. Il s'évanouit dans l'indicible. Mais un quatrième mouvement, normalement, devrait suivre. Normalement? C'est précisément la question. Les esquisses qui nous restent de ce final incomposé nous laissent deviner à quel point, pour Bruckner, il était alors incomposable. C'est comme si, le compositeur, avant tout le monde (avant les pressentiments de Mahler dans sa propre Neuvième; avant Schoenberg) avait découvert l'atonalité (Langevin, 1977). Les esquisses, si rudimentaires qu'elles soient, sont à cet égard indiscutables : une rupture inouïe s'impose au compositeur; il est au bord d'un gouffre, pris de vertige. Il s'arrête, alors, cherche à retrouver ses repères, 
son langage, ses assises, mais un territoire affolant, quoi qu'il fasse, s'ouvre devant lui. Et ce monde est si étrange, que la musique n'y ressemble plus à la musique.

Or Bruckner est âgé, fatigué, mal portant. Comment pourrait-il s'engager, seul, là où personne avant lui - même pas Wagner - ne s'est risqué? Comment pourrait-il admettre avoir conçu l'inconcevable? Comment donc, lui, fidèle à l'ordre tonal depuis toujours, serait-il en mesure de trahir ce qui lui tient le plus à cœur? Ou il trahit et alors il va jusqu'au bout de sa création. Ou il s'arrête et se trahit. Nouveau dilemme! Mais il y a autre chose, encore. Selon Langevin, la lecture des esquisses de ce final inabouti laisse deviner, chez Bruckner, une considérable crise morale. Tout au long de sa vie, il fut un fervent chrétien, d'une foi entière, têtue, proche de la bigoterie. Ce qu'il a mis en musique, dans ses Messes et ses Symphonies, c'est sa propre croyance. Il a bâti ses cathédrales de sons, élevé ses flèches, déployé ses rosaces. Et là, tout se brise! Ne demeurent que des décombres; que l'expression d'une désespérance absolue. Là où sa foi devrait culminer - en l'ultime mouvement de cette œuvre ultime -, c'est le doute qui ronge les assises. Une désillusion demeure, une décomposition de toutes les certitudes. La mort, donc.

Se risquera-t-il en cet insensé? Il l'esquisse, ce chaos, mais le creusera-t-il et l'ayant approfondi jusqu'aux dimensions d'un gouffre, fera-t-il de cette infernale descente, de cette damnation, les volutes de son dernier chant? S'il refuse de descendre, il se préserve, certes - mais ne préserve qu'une démission. S'il ne se préserve pas, il s'assume, certes - mais c'est comme un damné qu'il subsiste.

Bruckner s'arrête, en fin de compte. Il vit encore deux ans. Parfois, il revient aux esquisses. Il meurt à Vienne le 11 octobre 1896. "Dans la matinée, note Lucien Rebatet, il avait encore travaillé au final, qui resta inachevé, de sa Neuvième Symphonie» (Rebatet, 1969).

En 1909, Mahler achève la composition du Chant de la Terre. C'est là, comme au théâtre, le premier de trois coups successifs. Les deux autres? La Neuvième Symphonie puis le premier mouvement de la Dixième. Mais là, les trois coups n'annoncent pas le début de l'œuvre. C'est la fin du créateur - celle de toute une création qu'ils ponctuent par leur succession. Le rideau retombe sur le dénouement du spectacle. La musique se tait. Mahler n'est plus qu'un masque mortuaire. Immortel, cet homme hier encore si vivant? Sans doute. Mais dans quelques années, seulement. Du reste, Mahler, par boutade, posait la question: est-il vraiment nécessaire d'être vivant le jour où l'on devient immortel?
Le dernier mouvement du Chant de la Terre est une longue, très longue valse qui descend peu à peu, semble-t-il, vers des lieux infernaux. Tout est noir. Lugubre. Ensuite, vient une plage sereine mais, peut-on dire, d'une infernale sérénité. C'est là un "chant de la séparation et de l'évanouissement», écrit Bruno Walter (1969). Ce n'est pas la dernière œuvre que Mahler écrit, mais ce qu'elle exprime, cette œuvre, c'est qu'il y a une dernière fois! C'est qu'il est impossible qu'il n'y en ait pas. Respiration sourde d'un immense regret. Les derniers mots du Chant sont ceux d'un adieu: "Partout et éternellement bleuit l'horizon lointain, éternellement, éternellement...». En marge de la partition, en cet endroit précis, Mahler a noté nerveusement, à l'adresse des futurs chefs d'orchestre: «En mourant... ». Il faut interpréter ceci «en mourant».

En 1910, le compositeur met un point final à l'écriture de sa Neuvième Symphonie, travaillée simultanément au Chant de la Terre. Les deux œuvres se déploient en écho. Sans doute, le dernier mouvement de la Neuvième a-t-il été revu par le compositeur lors d'un de ses séjours à New York. Atmosphère étrange. Rapsodique. Océanique. Métropolitaine... Telle une arche lancée dans le vide, la détresse de l'homme tente de joindre l'autre rive de la vie. Mais en vain. C'est ainsi! Il faut l'admettre. Dans l'entre-deux, dans tout ce vide, il ne reste qu'à se laisser descendre au gré d'une disparition. La musique s'estompe. Elle tend vers le silence; vers une lumière de fin du jour. Ce qui s'estompe aussi, puis s'efface, c'est, avec la musique, l'homme lui-même: le compositeur. C'est maintenant la musique qui s'empare de lui. Elle l'emporte. En cette fin de partie, l'homme retrouve l'innocence de ses commencements. Tout fut dit. Tout pourrait encore se dire. Tout fut là. Tout ne pourrait-il pas l'être encore?

À propos de cette Neuvième, Schoenberg a noté: "Dans cette symphonie, l'auteur n'apparaît presque plus comme sujet. On dirait même que cette œuvre a été écrite par quelqu'un resté en coulisse, dont Mahler se serait simplement servi comme d'un porte-voix» (Schoenberg, 1977). Théâtre d'ombres, par conséquent. Un spectre de Mahler se tient déjà dans la pénombre, ailleurs, tout au fond de l'envers du décor, cependant qu'un autre Mahler - en pleine lumière, sous les feux de la rampe - lui confie l'écriture de cette page d'adieu.

Dans son étude: "Mahler avant la fin », le psychanalyste Théodor Reik, disciple de Freud, s'attache à saisir, chez le compositeur, cet instant où prend corps la Neuvième Symphonie (voir De La Grange, 1984). En cet instant-là, s'interroge Reik, quel fut le rapport de Mahler à l'idée de beauté? Quelle esthétique, donc, pouvait-il poursuivre encore en cette œuvre, sinon celle de l'extrême dépouillement? Quel sens le mot «plaire», pouvait-il conserver? Ni la quête de l'effet ni celle de l'apparat ni celle de la mise en spectacle ne le préoccupaient. Il éliminait tout cela; tout ce superflu. N'importait désormais, à la veille de sa mort, pensait-il, que la crucialité. Ne serait beau que ce qui serait vrai, même si cela fait mal. Parce que cela fait mal, précisément..

Cette vérité, donc, quelle était-elle si ce n'est celle du face à face avec l'inconnaissable? Pour le compositeur, en cet instant, exprimer l'inexprimable ne constituait pas un défi, uniquement, mais devenait une question vitale, véritablement, puisque c'est ainsi, par cet effort suprême, qu'il parviendrait à survivre à son propre anéantissement. «Dans la nudité, écrit Reik, il affronte enfin le néant et le désastre où quelque chose n'en finit pas d'essayer de se relever pour encore pleurer et encore mourir. »

En ce face à face, finalement, le compositeur saura-t-il se tenir debout? «Il ne regarde que droit devant lui, vers ce qui l'attend et qui est si proche au point d'être déjà présent, la fin, la terreur de la fin à laquelle il avait toujours songée et qui, pourtant, est absolument impensable, la mort. » Ainsi la beauté de l'œuvre n'a-t-elle plus de sens que si elle initie à une insupportable vérité cependant que la connaissance de celle-ci ne prend sens, elle-même, que si elle prédispose à l'adoption d'une attitude éthique: celle de la dignité humaine, face à l'inacceptable. Reik poursuit par ces mots : "Ici un homme s'élève au plus haut au moment même où il est écrasé. » Car la dissolution de l'être, maintenant, est là, toute proche. Elle attend sur le pas de la porte. Elle n'est plus théorique. "Maintenant, tout concept général de l'humanité est devenu inutile. Il s'agit de comprendre sa propre humanité, cette partie de soi qui est la plus humaine et cela avant de cesser d'être un être humain et de devenir une chose » (De La Grange, 1984).

Mais quelques mois plus tard, à l'été 1910, le compositeur est encore là, parmi les vivants. Diminué, mais vivant. À dire vrai, il se dédouble. Il y a le Mahler qui sauve la face, qui n'a pas le choix. Sa Huitième Symphonie, en effet - la plus monumentale de toutes ses œuvres - vient d'être créée. C'est un triomphe. La gloire du compositeur est désormais mondiale. Elle l'illumine. Il est en pleine lumière. Et cependant, il est dans l'ombre de plus en plus. Il y reflue. Y trouve refuge. Car il se sait perdu. Depuis quelques mois, il ne l'ignore plus: une maladie cardiaque va 
l'emporter. C'est sans remède. C'est alors qu'il apprend que sa femme le trompe avec un jeune et brillant architecte, Walter Gropius, promis à l'avenir que l'on sait. Qu'est-il donc, lui, Mahler? Que reste-t-il de lui? Qu'est-ce qui ne va pas en lui? Il va consulter Freud. Sans grand soulagement. Alors, que peut-il faire d'autre sinon entreprendre - puisqu'il vit! - l'écriture d'une nouvelle symphonie?

Ce sera la Dixième. Il ne l'achèvera pas. Seul est orchestré, achevé, le premier mouvement. Il laisse les trois autres en l'état d'esquisses, lorsqu'il meurt le 8 mai 1911. Il écrit. Écrit encore. "Ma vie n'aura été que du papier», confie-t-il, en un sourire crispé, à Alma, sa femme qui s'éloigne de lui. Tout s'éloigne, du reste, et jusqu'à sa propre raison. Il note rageusement entre les lignes de la partition: "Folie, prendsmoi, maudite sois-tu!»

Maudite mais désirée. Qu'il serait simple, en effet, de s'abandonner au délire! Alors, cette souffrance qu'il éprouve - si cruelle qu'elle soit - le détournerait de ce qu'il redoute plus que tout: sa décomposition. Il aurait le sentiment - délirant - de se maintenir en vie, malgré tout. Mais non! Il faut qu'il fasse face; tout s'en va, c'est incontestable. Et c'est bien ce que dit cette Dixième Symphonie, si inachevée qu'elle nous parvienne. Elle est une échappatoire entre une vie impossible et une mort insupportable. Une échappatoire vers une folie qui se refuse. C'est en toute conscience qu'il faudra, à Mahler, affronter sa fin.

Richard Strauss fut l'ami de Mahler. Il lui survit longtemps. En 1948, il a quatrevingt-quatre ans. Depuis des années, il ne compose plus. Pourquoi le ferait-il? Tout ce qu'il avait à dire, il l'a dit. Cela fait trop longtemps qu'il vit. Ses triomphes sont, pour lui, du passé déjà. Il y a eu la guerre. Des temps troubles. Il a fallu trouver des compromis : trouble, soi-même, parmi les troubles. Enfin! C'est ainsi. Et maintenant, ce qui pèse, c'est le poids de l'âge. Les maladies. Non, décidément, après Capriccio, après Les Métamorphoses, il ne peut aller plus loin. Il n'en a pas la force. Ni l'envie. Il se tait. Il ne s'occupe que de l'édition de ses œuvres d'autrefois.

Il n'est pas malheureux. Il est en attente. Mais l'attente n'en finit plus. Son fils, alors, le convainc de composer: d'écrire quelque chose, encore. Une dernière fois. Le vieil homme résiste. Puis d'un coup, en l'espace de quelques mois, compose quatre lieder. Derniers chants - sublimes - d'un homme qui ne chantait plus. En 1946, il écrit $A u$ crépuscule. Puis vient Printemps. Puis En allant dormir. Enfin, en septembre 1947 : Septembre.

Non pas une résignation à la mort, ces chants, mais un adieu à la vie. Strauss revient à une ligne mélodique simple, laissant cette écriture convulsive de beaucoup de ses œuvres passées. Simples, sereines sont ces réminiscences. On entend comme des souvenirs de voyages, comme les sonorités d'instants heureux. Une atmosphère italienne, méditerranéenne - a-t-on observé - imprègne ce final musical de sa vie. Un lyrisme retenu pour une mort en beauté. Mahler eut sans doute envié ce crépuscule de Strauss.

Une amputation annoncée... Ravel reçoit la commande, par le pianiste autrichien Wigenstein - amputé du bras droit d'un concerto écrit pour lui. Ce sera le Concerto en ré, dit: "Concerto pour la main gauche». Ravel achève de le composer en 1931. À partir de 1933, les premiers signes d'une très grave affectation cérébrale se font sentir. On parle d'" apraxie», de «dysphasie». Le fait est que le musicien éprouve, chaque jour plus, des difficultés à écrire, à bouger certains membres, à accomplir certains mouvements. Son cerveau se coupe de son corps. Son corps s'isole de son cerveau. Les centres nerveux qui mettent sa conscience en correspondance avec le monde extérieur sont détruits. Il se momifie vivant. Mais l'intelligence reste entière.

Bien sûr, il ne compose plus. Juste une œuvre mineure, après le Concerto en ré: une musique pour un film. Puis rien. Rien jusqu'en 1937, quand il meurt. Entretemps, il reste là, lucide. Il sait sa dégradation irréversible. "J'attends", dit-il en souriant à ses amis. On l'incite à voyager, tant que c'est possible. On l'accompagne au Maroc, en Espagne. Il voudrait écrire. Il a tout dans la tête. Rien ne sort de ses mains. On l'opère. Il ne survit pas.

1951. Los Angeles. Schoenberg attend sa fin. Loin de Vienne, loin de Berlin, seul, malade, presque pauvre, portant mal ses soixante-dix-sept ans, il compose encore cet opéra auquel il pense depuis longtemps - Moïse et Aaron -, qu'il laissera inachevé. Il se remémore. Ses premières œuvres. La Nuit transfigurée, les Gurre-Lieder, la Symphonie de chambre. C'est bien loin... C'était avant 1908. Il restait encore, alors, sous l'influence du romantisme allemand: Wagner, Bruckner, Mahler, Strauss. Puis il y avait eu la rupture. C'était lui, le premier, qui s'était aventuré sur ce continent inconnu de la musique: celui de la dissonance; de l'atonalité; d'une musique qui n'était plus faite pour plaire, pour être agréable aux oreilles, mais pour aller jusqu'aux limites extrêmes - parfois insupportables - de ses propres possibilités. Ç’avait été le temps de L'attente ou du Livre des jardins suspendus, mais surtout du Pierrot lunaire.
Il allait vers la quarantaine, à cette époque: l'âge de la maturité. Ce «parlé-chanté» qu'il introduisait dans son œuvre, ce qu'il attendait de lui, c'était qu'il questionne, lui également, les limites de la musique. C'était comme si elle voulait s'arracher à la parole, cette musique, pour y retomber malgré tout et s'arracher encore, mais en vain à nouveau. Tout ceci ne détruisait-il pas bien des choses entendues jusqu'alors? Il faudrait reconstruire, pourtant, un jour, de sorte que Schoenberg, quelques années plus tard, avait tenté d'y parvenir grâce à la découverte de la "série», de la discipline dodécaphonique. Il avait théorisé ce nouveau système d'écriture, mais composé des œuvres, également, mettant ce système en application. C'est alors qu'une autre rupture était intervenue: non plus en son œuvre, mais dans sa vie elle-même. Hitler avait pris le pouvoir en 1933 et Schoenberg, aussitôt, avait perdu son poste d'enseignant. Il avait donc quitté l'Allemagne, vers les États-Unis, vers la Californie. En même temps - par solidarité avec les siens, persécutés -, il était revenu au judaïsme dont il s'était éloigné jusqu'alors.

Le voici donc, à Los Angeles, composant cette œuvre dont il sait qu'elle sera la dernière: Moïse et Aaron. Depuis quelque temps déjà, il désire faire retour à des formes musicales plus classiques: aux fonctions tonales; aux harmonies du romantisme de sa jeunesse. Il ne renie rien de ces ruptures qu'il imposa à son art ni de ses novations ni de ses théories, mais il est las. Peut-on donc, sa vie entière, s'installer dans l'incommunicable, dans l'indicible, dans l'inouï, au nom d'une exigence supérieure - celle de sa quête transcendantale?

Et n'est-ce pas pour cela, précisément, qu'il compose cet opéra? Moïse, c'est un peu lui. Ou plutôt, c'est lui-même - songe Schoenberg - qui fut un peu Moïse. Pour pouvoir communiquer avec ce que la musique peut comporter de transcendant, d'ineffable, ne s'est-il pas coupé des hommes? De ce qu'ils sont en mesure de recevoir? Moïse avait gravi les pentes du mont Sinaï, se rendant vers l'inconnaissable, vers l'imprononçable. Aaron, en revanche, était resté parmi les autres, au milieu du peuple, à ras de sol, entre les hommes tels qu'ils se trouvent être, avec leurs désirs d'images, d'illusions, de plaisirs, de sensualité, de luxure. Lui, Aaron, à l'inverse de Moïse, savait fort bien communiquer. Il savait plaire. Séduire. Emporter l'adhésion.

Je fus Moïse, en somme, médite Schoenberg. Mais, en même temps, Aaron fut toujours là, en moi, comme en attente, disposé à se faire entendre. Aujourd'hui, nostalgique, comme je suis, de ces temps de tonalité - ne refusant qu'avec regret ces 
musiques de films qu'on me propose de composer -, n'est-ce pas lui que j'écoute? N'est-ce pas lui que j'entends, à nouveau, tout au fond de moi?

Schoenberg ne sait plus trop. Peut-être est-ce pour cela, précisément, que reste inachevé cet opéra. Cette inconclusion lui permet de ne pas choisir, de ne point devoir trancher, une fois pour toutes, entre Moïse et Aaron, entre ce versant et cet autre de son exigence musicale. Tout comme Moïse vieilli, incertain, Schoenberg, lui également, ne pourra guère fouler la terre promise de sa vérité révélée.

De Bach à Schoenberg, chaque musicien, en son temps, dans le climat de sa musique, s'affronte à la mort puis disparaît. Mais aussi, chaque musicien en poursuit un autre, de sorte que son prédécesseur ne meurt pas. Une continuité s'établit à travers ces ruptures. Une seule vie se poursuit - celle de la musique à travers la mort des musiciens. Et c'est comme s'il n'y avait, finalement, qu'un seul grand créateur poursuivant son œuvre par-delà les temps. Beethoven veut poursuivre Mozart qui veut prolonger Haydn. Schubert meurt en prononçant le nom de Beethoven. Mendhelson redécouvre Bach et se veut son continuateur. Schumann veut poursuivre Schubert. Brahms prend assise sur Schumann pour déployer son art. Bruckner, de Wagner à Mahler, établit un pont. Schoenberg, en dépit d'une rupture radicale qu'il introduit dans le langage de la musique, se reconnaît en l'œuvre de Mahler.

De sorte que des musiciens se taisent, chaque fois, mais que leurs voix trouvent des échos en d'autres, par la voie de subtiles métamorphoses. Chaque œuvre ultime, en ce sens, est aussi une prémonition; une annonciation. Elle clôt une vie, mais s'ouvre sur une autre. Ce qui nous reste, alors - ce que nous entendons, aujourd'hui -, ce sont ces moments suprêmes où la mort et la musique soudain ne font plus qu'un pour engendrer bientôt de nouvelles vies; de nouvelles formes musicales. Accouplements créateurs dont la musique, en son histoire, fait à la fois son tragique et sa vie, sa part d'ombre et de lumière.

C'est alors qu'il faut prendre conscience de cette difficulté: ces œuvres ultimes, ces compositions terminales dont nous avons parlé jusqu'ici, ne nous ont semblé exemplaires qu'en tant qu'elles s'avéraient exceptionnelles. Elles marquaient, condensaient, intensifiaient chaque fois - et selon les cas -, l'existence d'une crise, d'une tension tragique, d'un désir extrême, d'un pressentiment douloureux, d'une angoisse pathétique... C'est en cela qu'elles nous sollicitaient. C'est par cela - par cette hystérisation - qu'elles ont retenu notre attention: par l'effet de ces paroxysmes. Mais n'aurait-on pas dû parler, tout autant, de tous ces musiciens dont les morts ne furent rien qu'ordinaires et paisibles? Dont les œuvres terminales ne marquèrent en aucune façon un quelconque affrontement douloureux avec la mort?

À dire vrai, ce n'est point par complaisance ni par goût du tragique ou encore du morbide, qu'on s'est attaché, ici, à des cas particulièrement problématiques. C'est plutôt pour cette raison: les situations extrêmes sont précisément révélatrices de ce qui se trouve à l'œuvre en des situations plus communes, sans qu'on puisse le percevoir. Le tragique fait apparaître à vif - à fleur de peau - ce qui se masque, se cache sous les couches protectrices des circonstances plus ordinaires. Là, au contraire, tout craque; tout se met hors de soi; tout s'exhibe et se révèle. Nous saisissons, vivace, la question de la mort.

\section{AINSI LE COMPOSITEUR, S'ACHARNE-T-IL, ENCORE, À FAIRE VIVRE CE QUI POURTANT LE TUE.}

Le musicien peut-il prévoir l'instant où s'impose l'œuvre terminale? Peut-il savoir quand son heure est venue? Pas toujours; pas nécessairement. Parfois, il anticipe trop. Ainsi, de Schubert, composant La jeune fille et la mort de nombreuses années avant sa fin. Ainsi, encore, cette symphonie prémonitoire - la Huitième - laissée en l'état, par lui - avec deux mouvements, uniquement - alors qu'il composera par la suite la Neuvième Symphonie - dite «La Grande»- ainsi que plusieurs œuvres majeures de musique de chambre.

Parfois, en revanche, l'œuvre annonciatrice de la fin de toute chose n'a pas le temps de se réaliser, de se concevoir, que déjà le musicien n'est plus en mesure de lui donner jour. La maladie s'empare de lui. Elle le laisse impuissant. Ou encore, c'est la folie qui l'entraîne en des délires dont il ne sort que de vaines et tragiques tentatives de créer. Il arrive aussi que la stérilité, sans raison apparente, ferme ses serres sur l'esprit du compositeur. Il est là, disponible, lucide, et cependant, durant des mois, rien ne vient. Il n'avait pas prévu cette fin. Peut-être est-il mort à son œuvre, déjà, songe-t-il, alors qu'en apparence il vit. Car vit-on encore, existe-t-on, lorsque l'on ne vit que pour créer - et qu'on ne crée plus?

Tout à l'opposé, l'œuvre terminale, bien qu'elle mette un terme à la création, est voulue et pensée, elle-même, comme une création. Elle met un point final, certes, mais ce point, en un sens, est d'exclamation. De proclamation. Ici, proclame l'œuvre, s'arrête l'œuvre tout entière de ce musicien. Ici culmine la vague qui, déjà, par-dessous, se défait.

De sorte qu'ici, s'il le pouvait, le musicien voudrait tout mettre de lui: de ses désirs et de ses craintes; de ses aspirations, encore vivaces, et de ses lassitudes. Il aimerait que cette œuvre, à la fois, lui permette de passer vers la mort, mais qu'elle résiste à celle-ci. Il voudrait qu'elle sache dire, en son nom, adieu à l'existence, mais qu'elle s'en dépouille, pour le laisser nu face à lui-même - et à l'inexistence. Il souhaiterait encore que cette œuvre ultime sache le représenter à jamais - tel qu'il s'est accompli -, mais en même temps, cette composition, il voudrait qu'elle reste inachevée pour qu'il soit nécessaire qu'il revienne et l'achève.

Passage. L'œuvre ultime ressemble alors à un mouvement. Une transition. Elle s'entend comme un allant; un élan. À la fois porte de sortie hors de l'existence et porte d'entrée en l'inconnu de la mort, elle se tend vers l'inconcevable, l'inouï, l'infigurable. La composant, le musicien se construit une nef, donc. Il y embarque pour transmigrer. Elle le transporte. Un jour, en effet, n'a-t-il pas pressenti que la fin approche? Il veut se ménager une méditation. Sa musique l'abritera, le portera, songe-t-il. C'est à sa façon qu'il accomplira le voyage - créateur encore, bien que presque détruit - avant que la décrépitude ne le contraigne à un périple qui lui échappe.

Résistance. L'œuvre est là, en ce cas, pour contrarier la mort; pour la retarder. Elle prend la forme d'un refus. Elle est un écran que le musicien interpose entre ce qu'il est encore aujourd'hui - créateur et vivant - et ce que demain lui promet de devenir. Pour cela, il récapitule; tente de composer une œuvre terminale qui sache contenir tout ce qu'il aura tenté, tout au long de sa vie, de mettre au monde. Ses grands thèmes. Ses découvertes. En ce sens, l'œuvre terminale prend figure de défi: tout se décomposera, bientôt, en ce créateur condamné à la mort, mais pour l'heure tout se compose si magnifiquement, si magistralement, que la mort n'a qu'à bien se tenir. Qu'elle se le tienne pour dit: le créateur n'a pas dit son dernier mot; n'a pas chanté son dernier chant.

Nostalgie. Ce que le musicien récapitule, ce ne sont pas uniquement, en son œuvre terminale, ces trouvailles qui firent son génie. Ce sont aussi ses souvenirs, ses amours, ses souffrances, ses joies. Que cette vie fut belle, si abrupte qu'elle soit! Éternellement, éternellement, elle res- 
tera tandis que le musicien disparaît. Et donc, tout ce qui s'évanouit déjà, tout ce qui s'efface, le compositeur ne peut-il le tenir, un instant encore, dans les mailles de sa partition? L'œuvre n'est alors pas un mouvement. Elle est un moment: un instant d'exception. Elle ne résiste pas, mais s'attarde. Ce qu'elle voudrait tenir en elle, maintenir à jamais, ce sont ces sonorités d'un passé. Tout ne fut pas heureux, certes, en l'existence du musicien. Mais il fut heureux que cela soit.

Abandon. L'œuvre terminale cherche donc à retenir; à sédimenter; à fixer à demeure. Mais en même temps, elle s'abandonne. Elle se délite. Qu'y a-t-il à perdre, à reconnaître que tout s'en va? Tout est perdu, de toute façon! Autant l'admettre; autant le dire et faire de cette reconnaissance quelque chose qui ne se perdra pas; qui restera à jamais comme la trace d'une inévitable disparition. L'œuvre se dépouille. Elle veut aller à l'essentiel. Oublier l'apparaître et les déductions. Oublier de «prendre les formes». Ne reculer devant rien et, surtout pas, devant les convenances. Hors de toute précaution, loin des sentiers battus, l'œuvre ultime s'aventure alors en des déserts qui lui ressemblent.

Perdurance. Cette œuvre complexe qui vient accompagner la fin d'une existence cette œuvre faite d'abandon, de nostalgie, de mouvance, de résistance - survivrat-elle au musicien? Il le voudrait, certes. Il aimerait qu'elle subsiste, présente, comme le témoignage de ce qu'il fut au monde, luimême, et jusqu'au dernier souffle de son inspiration, s'obstina à créer. Ses autres œuvres sont derrière lui. Elles constituent son patrimoine. Celle-ci, au contraire, il la lance tout devant, il ne sait où, pour qu'elle l'attende plus loin et qu'il puisse la rejoindre. Ainsi se confondront-ils, elle et lui, en une même postérité. Cette présence que je mets en mon œuvre, médite le musicien, sera là, encore - sera présente, parlante, audible - quand je ne serai plus. En ce sens, je serai là. J'y serai plus encore, sans doute - encore plus intensément que je ne puis être présent, aujourd'hui, en ce semblant de vie qui me reste. Elle me survivra. Je m'y survivrai.

Inachèvement. L'œuvre, donc, survivrat-elle à son auteur? Peut-être lui survivra-t-elle d'autant mieux qu'elle demeurera inachevée. Lui-même, bientôt, ne sera plus: il sera achevé par les ans et l'usure. Elle-même, non finie, laissée comme en suspens, restera à jamais dans l'attente de se clore. Jusqu'où serait-elle allée? Vers quelle culmination? Vers quelle chute? Il faudrait que le compositeur revienne pour nous le dire et peut-être - laissant ainsi son œuvre inachevée - a-t-il espéré (consciemment ou non) qu'il soit nécessaire qu'il revienne. Aurait-il bouclé la boucle, il ne serait plus indispensable. Ne l'ayant point parachevée, c'est comme s'il suspendait, jusqu'à la fin des temps, le couperet de son propre achèvement.

Ne pas pouvoir aller plus loin... Aller, si on le peut, aussi loin que l'on peut dans l'écriture de l'œuvre terminale afin de dire que non, décidément, on n'ira pas plus loin. Cela s'achève, une fois pour toutes. Ainsi le compositeur, s'acharne-t-il, encore, à faire vivre ce qui pourtant le tue. Mais l'interprète? Lui dont, jusqu'ici, rien n'a été dit? Lui, à son tour - chef d'orchestre ou soliste -, un siècle plus tard, reprendra l'œuvre terminale. Il la relancera, alors, en un présent que le compositeur, bien entendu, ne peut connaître. Grâce à l'interprète, son œuvre, elle, connaîtra ce présent.

Mais un jour, cet interprète - créateur de la vie d'une œuvre annonçant une mort - connaîtra la mort, lui-même. Du moins, la sentira-t-il approcher, s'éprouvant vieillir. Il ne sera plus maître de son art, comme autrefois. Son corps le trahira. Son esprit, également, qui saura moins retenir toutes les subtilités d'une partition. Ne sera-t-il pas temps qu'il s'arrête? Avant qu'il redescende, ne lui faudra-t-il pas savoir s'en tenir au plus haut; en rester à ce point ultime où il s'est rendu ?

Ultime, sublime. Terminal, carcéral. Par-delà leur sonorité - par-delà leur musique -, il semble que ces termes soient en correspondance. L'ultime est le plus loin. Impossible, en effet, de se rendre audelà de l'ultime sans rencontrer, précisément, l'inconcevable et le néant. Quant au sublime, qu'est-il d'autre, si ce n'est le plus haut? Impossible de s'élever par-delà le sublime. Impossible sans se risquer à rencontrer le divin. Souvenons-nous: «Je vais enfin entendre la véritable musique», disait Bach sur son lit de mort. Il n'irait pas plus loin. Il n'irait pas plus haut. Mais plus loin et plus haut, peut-être, entendrait-il - ultime et sublime - la musique de la Création.

Tensions aux extrêmes, donc. La première tension, celle qui porte vers l'ultime, est imposée à l'homme: elle est celle de la mort. Face à elle, il n'a pas le choix. Il n'ira pas plus loin. Son temps va s'arrêter. Mais la seconde tension, elle - celle qui porte au sublime - relève de sa liberté. S'il le veut, il peut surenchérir, opposant à sa propre destruction la création de l'œuvre; opposant à la tyrannie de l'ultime la musique du sublime.

Ainsi l'homme, par la grâce de l'œuvre - par sa quête du sublime - affirme-t-il son humanité à l'encontre de l'ultime - de la mort - qui est le sort de l'humanité. Mais le mouvement peut être autre, dialectisant cette fois ces deux autres termes: terminal et carcéral. Terminal, en effet, est en quelque sorte de l'ultime malheureux. La vie du créateur se termine mal. Elle se délite dans la folie, l'angoisse ou la stérilité. Le surenchérissement du créateur, en ce cas, se renverse. Il ne monte plus vers le sublime. Il ne cherche plus, se hissant, à dépasser l'indépassable, mais descend vers l'insoutenable au contraire, vers un chaos insupportable dans le but de s'y enfermer. C'est Goya vieillissant jetant les figures de ses monstres sur les murs de sa maison, dont il ne sort plus. C'est Schumann tournant en rond, jusqu'au vertige, dans les boucles de ses Variations fantômes. C'est Beethoven poursuivant sa musique dans l'enclos de sa surdité. C'est Nietzsche, encore, à l'asile, n'écrivant plus rien qui fasse sens.

Les musiciens, face à la mort, sont les frères de tous ces créateurs - peintres, écrivains, cinéastes, chorégraphes, comédiens - qui, eux-mêmes, par la voie de leur œuvre, sont contraints de s'affronter à la grande inconnue. Les uns comme les autres, par leur art, ont fait acte de présence en ce monde où ils sont passés. Ils ont opposé à l'inhumanité de leur future absence - que l'on nomme : la mort -, l'humanité de leur éphémère passage. Nous écoutons, contemplons, déchiffrons les traces de ces passages. Ce sont leurs œuvres. Nous les suivons, ces œuvres, jusqu'à ce que, soudain, nous voyons qu'elles s'arrêtent. Plus rien après. Rien, si ce n'est notre propre pensée sur les dissonances de la fin.

\section{Bibliographie}

BOUCOURECHLIEV, André (1991). Essai sur Beethoven, Paris, Actes Sud.

DE LA GRANGE, Henry-Louis (1984). Gustave Mahler. Tome 3. Le génie foudroyé, Paris, Fayard.

LANGEVIN, Pierre-Georges (1977). Bruckner. Apogée de la symphonie, Lausanne, L'Âge d'homme.

REBATET, Lucien (1969). Une histoire de la musique, Paris, Robert Laffont, coll. «Bouquins».

SCHNEIDER, Michel (1989). La tombée du jour. Schummann, Paris, Seuil.

SCHOENBERG, Arnold (1977). Le style et l'idée, Paris, Buchet/Chastel.

WALTER, Bruno (1969). Gustav Mahler, Paris, Livre de poche, Pluriel. 